Journal of Theoretical and Applied Mechanics, Sofia, 2016, vol. 46, No. 2, pp. 51-64

\title{
AUTOMATIC BONE DRILLING - MORE PRECISE, RELIABLE AND SAFE MANIPULATION IN THE ORTHOPAEDIC SURGERY*
}

\author{
George Boiadjiev \\ Faculty of Mathematics and Informatics, Sofia University, \\ 5 J. Boucher Blvd, 1164 Sofia, Bulgaria \\ Institute of Mechanics, Bulgarian Academy of Sciences, \\ Acad. G. Bonchev, blok 4, 1113 Sofia, Bulgaria \\ e-mail: george@imbm.bas.bg \\ RUMEN KASTELOV \\ Orthopaedic and Trauma Clinical Centre of Ministry of Domestic Affairs, \\ 79 Skobelev Blvd, 1606 Sofia, Bulgaria \\ e-mail: rkastelov@gmal.com \\ TONY BOIADJIEV \\ Institute of Information and Communication Technologies \\ Bulgarian Academy of Sciences, \\ Acad. G. Bonchev St., Bl. 2, 1113 Sofia, Bulgaria \\ e-mail: tonyboiadjiev@mail.bg \\ Kamen DeLCheV \\ Institute of Mechanics, Bulgarian Academy of Sciences, \\ Acad. G. Bonchev St., Bl. 4, 1113 Sofia, Bulgaria \\ e-mail: kamen@imbm.bas.bg \\ KAZIMIR ZAGURSKI \\ Institute of System Engineering and Robotics \\ Bulgarian Academy of Sciences, \\ Acad. G. Bonchev St., Bl. 2, 1113 Sofia, Bulgaria \\ e-mail : kazz@mail.bg \\ [Received 15 April 2016. Accepted 20 June 2016]
}

ABstract. Bone drilling manipulation often occurs in the orthopaedic surgery. By statistics, nowadays, about one million people only in Europe need such an operation every year, where bone implants are inserted. Almost always, the drilling is performed handily, which cannot avoid the

\footnotetext{
${ }^{*}$ Corresponding author e-mail: george@imbm.bas.bg.
} 
subjective factor influence. The question of subjective factor reduction has its answer - automatic bone drilling.

The specific features and problems of orthopaedic drilling manipulation are considered in this work. The automatic drilling is presented according the possibilities of robotized system Orthopaedic Drilling Robot (ODRO) for assuring the manipulation accuracy, precision, reliability and safety.

KEY WORDS: Automatic bone drilling, orthopedic surgery, accuracy, precision, safety, control.

\section{Introduction}

Orthopedic screws are often implanted in the bones. That needs to realize the bone drilling process before. Until now, the bone drilling mostly is executed by hand in the orthopedic surgery. But hand-drilling leads to some problems as getting the big outlets - by reports the average error in bi-cortical drilling manipulations is $6.31 \mathrm{~mm}$ ! [1], also, breaking the tendons or blood vessels, protecting the rear bone wall (which brings additional cutting of the tissue), overheating and so on [2-4]. Such problems concern the precision and accuracy of the drilled holes.

The main factor causing the problems mentioned above is the variable resistant force at drilling. It is for the sake of non-homogeneous density of bones and their structure (tube and soft one), as well as the various patient state (age, sex, health status etc.). In the same time, the manipulation safety entirely depends on the surgeon skill, i. e. his subjective behaviour. Making the orthopedic operations automatic ones, there, many problems can be solved just by decreasing the subjective factor influence. Moreover, that can assure better accuracy, precision and reliability of the manipulation.

The first application of robots in the orthopedic surgery is based on the industrial robots-manipulators. The advanced tendency in that field is oriented to a design of manipulative systems, according to the specifics of concrete orthopedic manipulations [5-7]. Some robotized systems are developed aiming to perform specific orthopaedic manipulations automatically [7]. Among them, RoboDoc (US), Acrobot (UK), MARS, CRIGOS, CASPAR etc., are well known [7].

The main requirement is assuring a maximal reliability, taking into account that the objects under manipulation are the human being organs. The last leads to the conclusion that the robot usage in surgery must be such ones designed especially for specific operation, only $[7,8]$. Such an approach allows assuring: 
- maximal simplifying the robot mechanical system and minimizing its degrees of freedom;

- level of applied force action according to any specific case;

- sensor system and software simple enough to allow the surgeon an effective control of the robot executed manipulation.

In the work, the accuracy and the safety problems in the automatic bone drilling are discussed, as well as their way of solution by application of robot system (ODRO) $[9,10]$. The components of ODRO, its mechanical construction, electronics, program environment and language, as well as software are published in [11-17].

The object of the work is to show that the automatic bone drilling guarantees more precision, reliability and safety of bone drilling manipulation and in this way, improves the result quality.

\section{Robotized system for bone drilling (ODRO)}

Developing a research on automatic bone drilling in surgery different experimental setups were designed and many experiments were executed [1117]. As a result, the drilling process parameters were investigated and the criteria were formulated, which an automatic drill system is supposed to answer.

In Fig. 1, the last prototype of automatic drill machine ODRO is presented, as well as its accessories by which hard connection between the bone and the executive device corpse is realized. Robotized systems control block is shown in Fig. 2.

The bone drilling robot maintains two working modes:

- Handily;

- Automatic.

The machine is working as usual, drilling one in handily mode. The rotation speed is possible to be regulated by potentiometer.

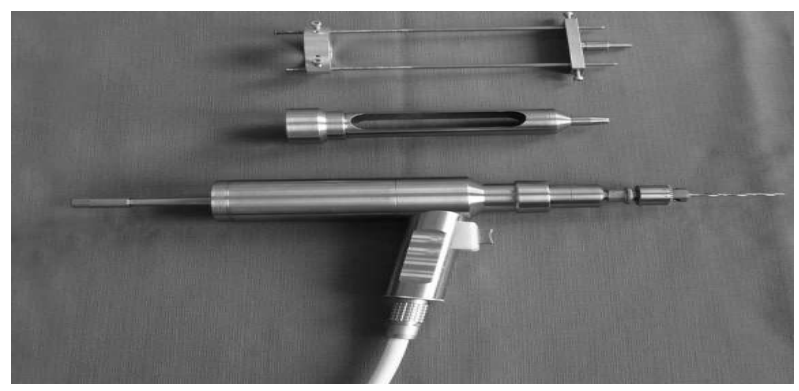

Fig. 1. Automatic drilling executive device and its accessories 


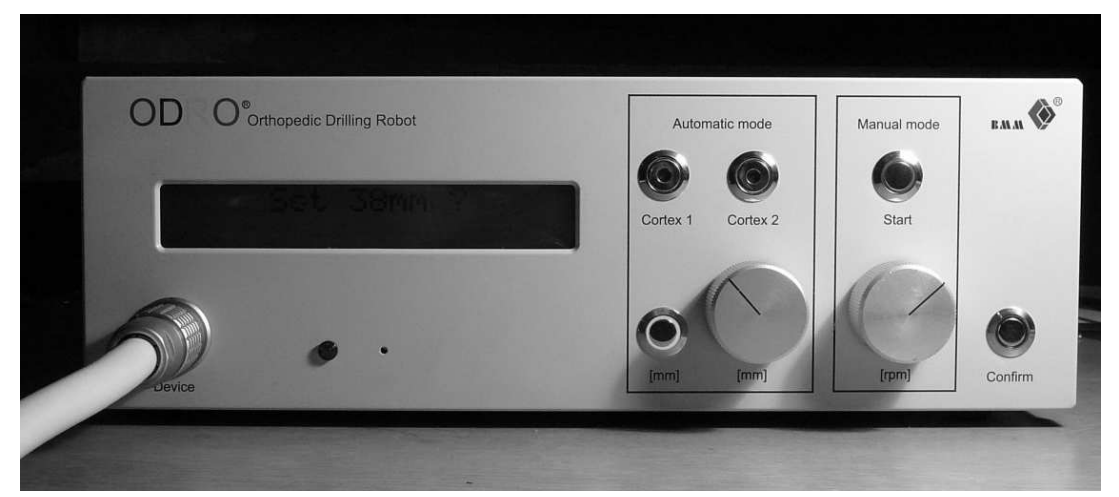

Fig. 2. Control block

The automatic mode has two sub modes:

- entirely drilling the bone (Cortex I, Cortex II);

- drilling the preliminary set depth ( $\mathrm{mm})$.

Submodes are chosen by the surgeon with the help of four buttons and potentiometer connected with display.

\section{Accuracy of the manipulation}

Depth is required when drilling the preliminary set. It is given by $1 \mathrm{~mm}$ step within the interval from 2 to $100 \mathrm{~mm}$. Such step is in correspondence with the orthopaedic screws used in the orthopaedic surgery and available in the market.

The manipulation accuracy means that the preliminary set depth is realized with some deviation acceptable in surgery practice. This 'error' is placed as a parameter in the software program and in our case it is $0.1 \mathrm{~mm}$. The 'Contact Position' value is registered, after realizing the drill bit contact with the bone. It is used as a basement (initial value) for preliminary set depth drilling execution. When the preliminary set depth is reached, the drilling stops and the procedure for verification is executed, because the translation motion by step motor may cause a probability of missed steps during drilling process. The last statement means that the depth shown by controller really may be less than the needed one. The accuracy check for real depth is performed in following way:

- after drilling end the translation module goes back to the Reference Position, while the actual position is keeping continuously in the memory; 
- during drilling with $0.1 \mathrm{~mm}$ error, which is equivalent to 403 steps, the actual position decreasing is compared with 403 and in the same time the Reference Switch is taken into account. The linear drive works in 64 microsteps/step mode which is related to $4032 \mathrm{step} / \mathrm{mm}$;

- reaching the actual position values less than 403 and in the same time, the Reference Switch stays unchanged. That means, if existing steps there are missing, then their number is less than 403, during translation motion execution;

- the program lifts its flag, which shows that the preliminary set depth is realized with corresponding accuracy.

The message on the display, shown in Fig. 3, gives information for preliminary set $22 \mathrm{~mm}$ depth drilling and real drilled depth after the check procedure.

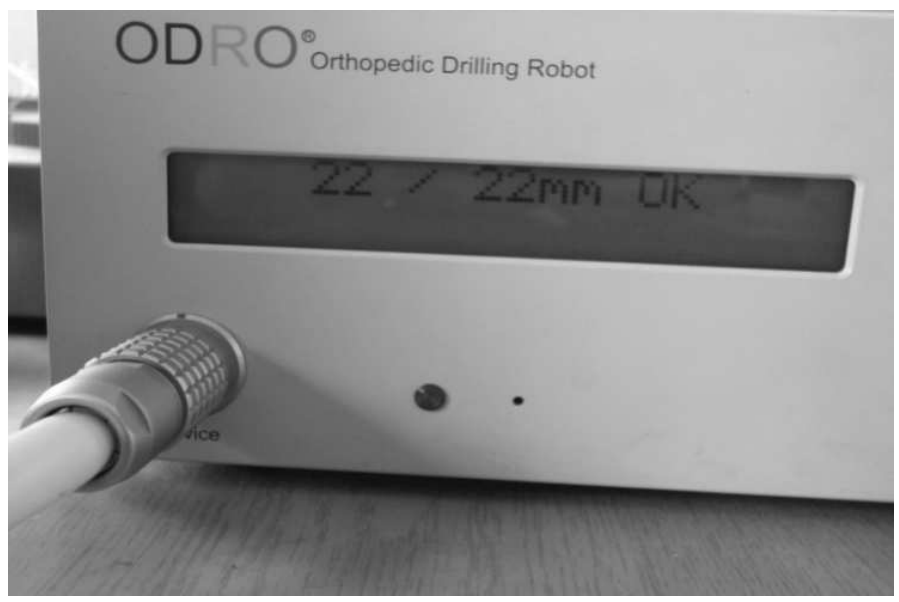

Fig. 3. Confirmation message after preliminary set depth drilling of $22 \mathrm{~mm}$

Practically, the preliminary set depth drilling with $0.1 \mathrm{~mm}$ accuracy is not wide spread, because the usually used orthopaedic screws have steps 2 $\mathrm{mm}$. Nevertheless, some manipulations need more precision, as spine column drilling, for instance [16]. The control algorithm, motor and controller used here give a possibility to perform the manipulation with such accuracy.

\section{Manipulation precision}

The manipulation precision is related mainly with the registration of moment of time, when the drilling ends. According to the chosen drilling mode, the end task decision concerns achievement of the preliminary given depth or 
registration of the far cortex end, when the bone is drilled entirely. In the last case, several parameters are processed by corresponding algorithm. The bone resistant force $F_{\text {Act }}$ is monitored continuously during the drilling process. The difference $\varepsilon$ is formed for every discrete sample $s$ :

$$
\varepsilon=F_{A c t}-F_{R},
$$

where $F_{R}$ is resistant force, which characterizes the concrete object of manipulation.

On that base, an integral component is formulated:

$$
I_{e r r}=\sum_{s=1}^{4} \varepsilon_{s},
$$

which is 'flow window', having 4 samples of discrete dimension. That component gives information for resistant force deviation round the drilling.

The decision to finish the bone drilling ('Stop Decision') is taken as a function of following parameters $-F_{0}$ (free motion resistance), $F_{R}, F_{A c t}$ and $I_{\text {err }}$. Also, taking into account additional criteria, the probability of closing to the bone end is evaluated and the translation speed is reduced to $1 \mathrm{~mm} / \mathrm{s}$. The last operation aims to assure minimal penetration out of the bone and not to cause some debris when the breakthrough occurs.

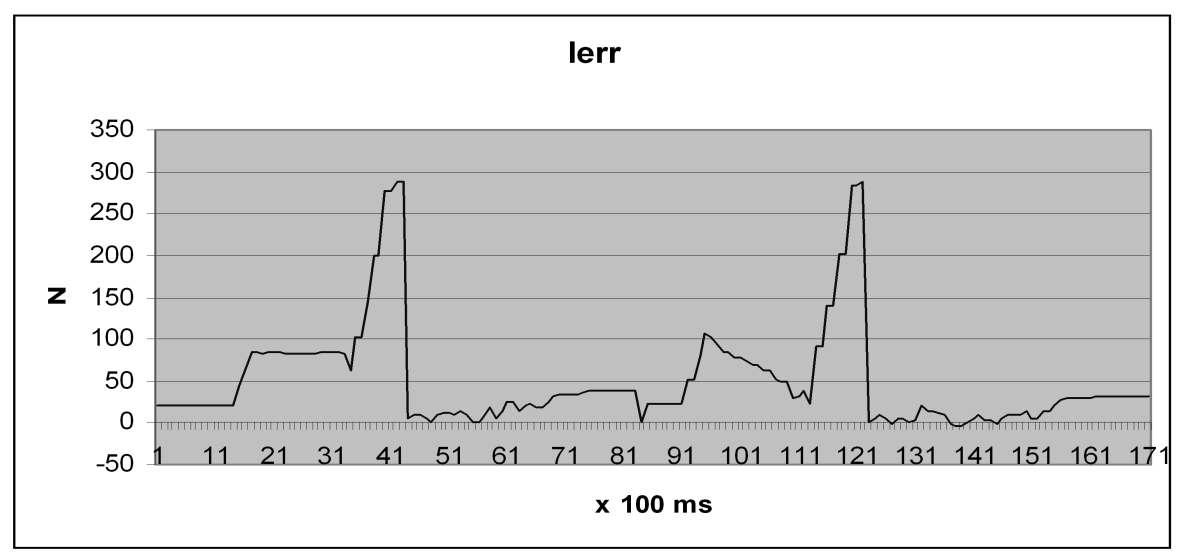

Fig. 4. Deviation of $I_{e r r}$ in entirely pork bone drilling mode with $2 \mathrm{~mm}$ drill bit diameter

Experimental data are presented in Fig. 4 for deviation of $I_{e r r}$ with respect to time during drilling of pork bone, entirely. Every measurement is recorded by $100 \mathrm{~ms}$. 
Time is scaled on the horizontal axis ( $x$-axis), as conditional points $(\mathrm{CP})$. The real time interval is obtained multiplying CP interval by $100 \mathrm{~ms}$. From the graphics, the following zones can be seen and they are commented as:

- searching contact with the first bone cortex: up to $34 \mathrm{CP}$;

- forming a center and resistant force identification: 35-44 CP;

- first cortex drilling: up to $74 \mathrm{CP}$;

- decision for closing the bone end and speed reduction to $1 \mathrm{~mm} / \mathrm{s}: 69-70$ $\mathrm{CP}$;

- first cortex drilling and decision for first cortex drilling end: 71-73 CP;

- stop the motors: $74 \mathrm{CP}$;

- motion start again throw marrow: from $74 \mathrm{CP}$;

- searching contact with the far bone cortex: until $113 \mathrm{CP}$;

- forming a center and resistant force identification: 114-123 CP;

- far cortex drilling: up to $157 \mathrm{CP}$;

- decision for closing the bone end and speed reduction to $1 \mathrm{~mm} / \mathrm{s}: 157-158$ $\mathrm{CP}$;

- far cortex drilling and decision for far cortex drilling end: 159-161 CP;

- stop the motors: $162 \mathrm{CP}$;

- going back to Reference Position after 163 CP.

The whole drilling time is about $16 \mathrm{~s}$. The first cortex is drilling (from contact registration until the drilling end) for $4 \mathrm{~s}$ and the far cortex - for $5 \mathrm{~s}$. The time for registration the far cortex end and the end of drilling is $5 \mathrm{CP}$. The drill bit penetration outside the far cortex is about $0.7-1 \mathrm{~mm}$, taking into account the drilling speed.

The speed reduction to $1 \mathrm{~mm} / \mathrm{s}$ at the drilling end allows forming the breakthrough very precisely, without bone debris. But minimization of diameter variation along the drilling hole is connected with translation orientation maintenance, which is responsibility of the surgeon (as it is for position contact maintenance).

An across cut section after whole drilling of pork bone is shown in Fig. 5. No variation of the hole along the depth can be seen.

\section{Manipulation safety}

Patient safety is the most important requirement in bone drilling manipulation. That means, the control algorithm creation must be such one to exclude the bone and the soft tissue damages. 


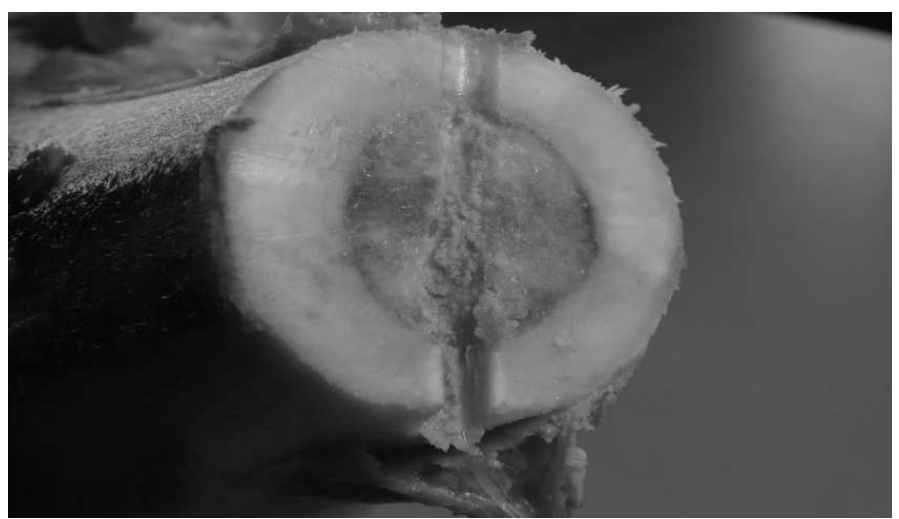

Fig. 5. Pork bone across cut section, after whole drilling with $6 \mathrm{~mm}$ drill bit diameter

The successful manipulation realization depends on the normal functioning of the system components - motors, force sensor, controllers, buttons, potentiometers, etc. Or the components must be tested before manipulation itself. For that purpose, a procedure 'Self Test' is developed, which starts immediately after the machine power is switched on. During the 'Self Test' procedure, the following testing steps are executed:

- start button reliability (switch on and switch off);

- find out the initial position (Reference position);

- force sensor;

- translation motion performed by step motor (going forth and back the working zone, free motion resistance, check for missing steps);

- rotation motion.

The force sensor test includes resistant force calculation at free translation motion forth and back and an average value is obtained, which is a criterion for normal motion.

The decision for right or wrong working component is taken, according to criteria, built in the program. The 'Self Test' procedure allows working of all the system, if the main components work correct. When some deviation from the built in criteria is registered, then the message "Self TEST ERR" appears on the display and the machine cannot be used, until the reasons for that are eliminated. The message on the display after positive result of the procedure is seen in Fig. 6.

The thrust force control during the drilling process is performed by translation motion control. It is realized by setting the concrete values for specific controller parameters, which define the maximal translation speed, max- 


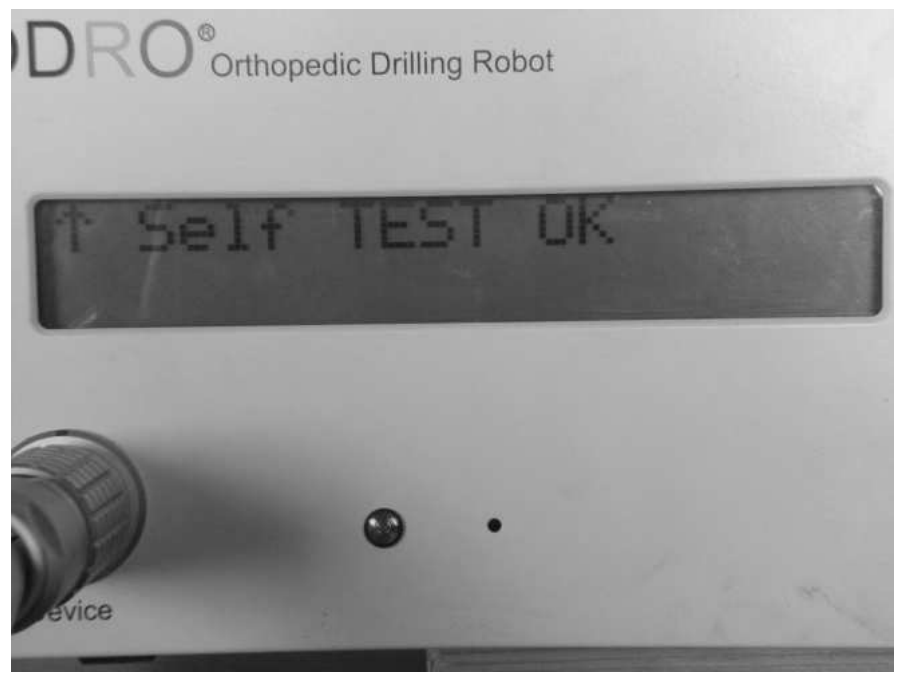

Fig. 6. The message on the display after positive result of the procedure 'Self Test'

imal acceleration, as well as target position in correspondence of the current position.

It is executed very slowly in $1 \mathrm{~mm}$ distance with $0.5 \mathrm{~mm} / \mathrm{s}$ speed at the drilling beginning, after the contact registration with the bone. In this way, a 'center' is formed aiming to eliminate the drill bit slide on the bone. That is very important in the cases when the drill bit is not oriented perpendicular to the bone. The next $1 \mathrm{~mm}$ is drilling with $2 \mathrm{~mm} / \mathrm{s}$. During that depth, realizing bone identification is doing, which bone itself is concrete object of current manipulation. The result is a concrete value, which characterizes the bone density. That is achieved by taking average data of the force sensor, which data are recorded in every discrete sample time. The identification purpose is used for the obtained value to evaluate the applied thrust force in correspondence with the concrete object and in this way to avoid the additional trauma risk.

Algorithm for target position calculation is created in dependence of arising resistant force. Its purpose is minimizing the difference between identified resistant force, which characterizes the concrete object and the actual resistant force, measured by the force sensor during the drilling process execution. Maintaining this difference in the borders close to zero means control of thrust force aiming to keep values close to the resistant force, which characterizes the concrete object under manipulation. The algorithm is a classical PI control low, where the proportional component is given by equation (1) and the integral one - by (2). The feedback coefficients $k_{p}$ and $k_{i}$ are tuned 
experimentally.

The machine can perform any motion, only when the surgeon keeps on the start button pressed. Any motion stops immediately, if the button is released. When the button is pressed again, the machine goes on manipulation (program) execution from the moment of the time, when the motion has been broken. By the surgeon evaluation, the current manipulation can be finished and the new task (new working mode) can be set without system restarting. The information seen on the display in real time shows what part of the task is executed up to the current moment. In that sense, the surgeon can control the drilling process in real time. In Fig. 7, the display shows the moment of realizing the depth of $4 \mathrm{~mm}$, when the preliminary depth is set as $11 \mathrm{~mm}$.

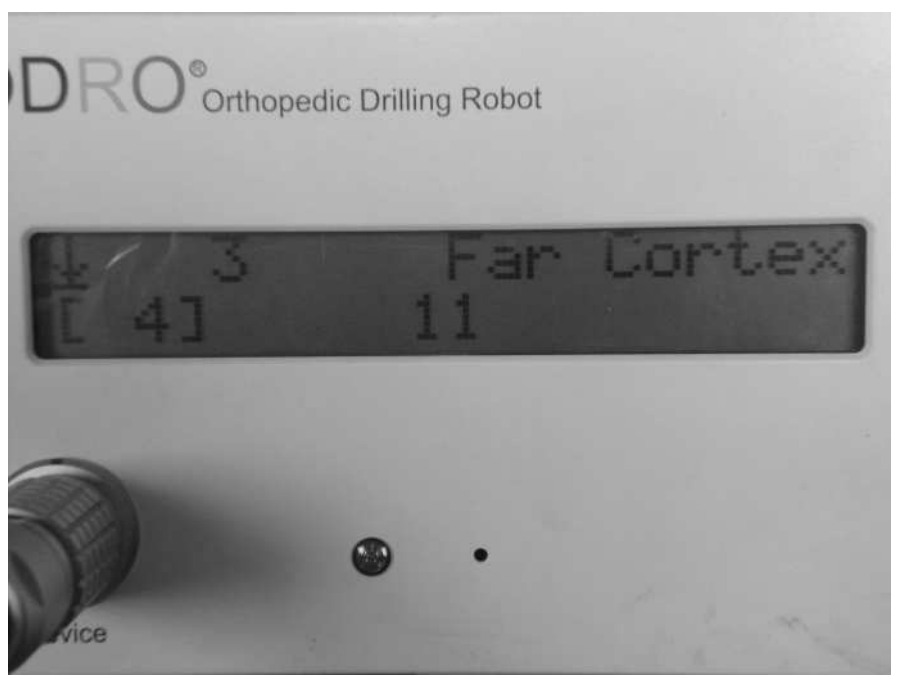

Fig. 7. Current real time information on the display

The subjective evaluation of the surgeon is not possible to be avoided and it is not desirable at all. He must specify the working mode, the necessary orthopaedic drill bit, as well as the desired its maximal penetration. The system functions are just to keep the chosen parameters with needed accuracy and to guarantee the critical parameters will never be exceeded.

It is predicted when no correspondence between set parameters and real conditions exists, then the system displays warning messages.

For example, if the set depth is longer than the available (short) drill bit, the warning message displays as "Error Prop", i. e. it is impossible to finish the operation with such a drill bit (Fig. 8). Of course, this check is operating after the contact detection - then the machine goes back to initial position. 


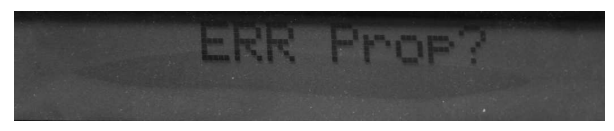

Fig. 8. The working zone exceeding

Another example is when the machine works in whole bone drilling mode (Cortex I and Cortex II). Then, two parameters are set: number of cortexes and maximal hole depth (Fig. 9).

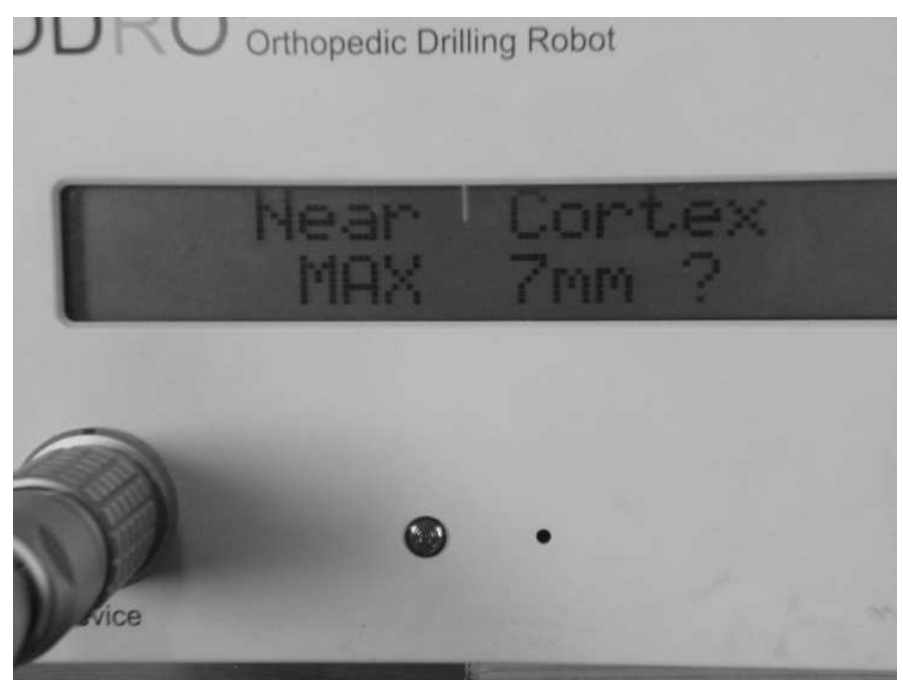

Fig. 9. Setting the working mode

In this case, the main aim is the second parameter - maximal depth MAX mm. This way, it is guaranteed if some error occurs or the far cortex end is determined wrongly, then the patient will not be damaged, because the drill bit will not appear after the bone end and will not break the arteries, tendons and soft tissues close to the far cortex end. As a comparison, the traditional drilling depends on the surgeon skills only.

The surgeon himself decides whether some drill bits can be used according their sharpness. But if drill bits are not sharpened enough (for the sake of long usage with), this leads to increase of the drilling time and the temperature, i. e. the risk of the bone damage. For that reason, the drilling process is continuously controlled from the point of view of reached depth for unit time. The last is as a criterion for the extent of the drill bit sharpness. If not sharpened enough is used, the display shows warning message together with noise signal 
(Fig. 10). In this case, the manipulation does not break automatically - the decision is in the surgeon hands.

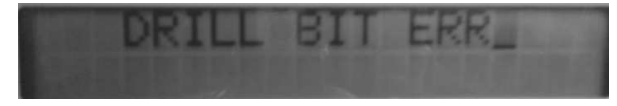

Fig. 10. Warning message for not enough sharpened drill bit

After the whole bone drilling, the following information can be seen in the display (Fig. 11):

- first cortex thickness;

- distance between both cortexes (marrow thickness);

- far cortex thickness;

- the whole depth measurement.

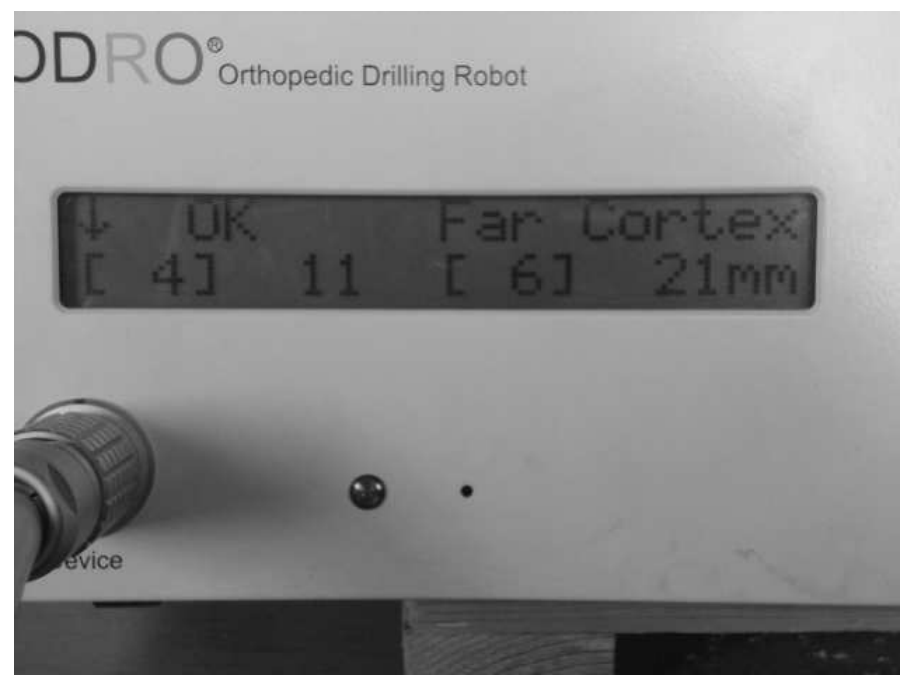

Fig. 11. Information after the whole drilling end Near cortex $-4 \mathrm{~mm}$; Marrow - $11 \mathrm{~mm}$; Far cortex $-6 \mathrm{~mm}$; Total $-21 \mathrm{~mm}$

In real operation, the information shown in Fig. 10 has very important meaning - it eliminates another whole manipulation: measurement of already reached depth. The measurement in the concrete case is performed with 1 $\mathrm{mm}$ accuracy. Even though, the system capability is higher $-4932 \mathrm{steps} / \mathrm{mm}$. But from practical point of view (except the spine column operation [16]), the existing machine accuracy is enough, because the screw has steps of $2 \mathrm{~mm}$. The information in Fig. 10 is also important in the sense of optimal choosing the implant screw length. 


\section{Conclusion}

The present paper pays attention to very important characteristics of the bone drilling manipulation in the orthopaedic surgery - accuracy, precision, reliability and safety. During automatic bone drilling the accuracy is $0.1 \mathrm{~mm}$, which is due to the linear drive capabilities and the control algorithm.

The precision is assured by control algorithm software realization. Before the start of manipulation, as well as during its execution, the tests and checks are carried out in correspondence with the downloaded in the software program criteria. That is oriented to assure the manipulation safety.

The display, which shows real time information gives additional possibility to the surgeon to control the operation and to take decision in dependence of the concrete situation.

Generally concluding: the automatic bone drilling assures various advantages and can solve the problems, existing in usually drilling manipulation execution by hand.

\section{REFERENCES}

[1] Clement, H., N. Heidari, W. Gechenig, A. M. Weinberg, W. PichLER. Drilling, not a Benign Procedure: Laboratory Simulation of True Drilling Depth, Injury. Int. J. Care Injured, 43 (1012), No. 6, 950-952, doi:10.1016/j.injury.2011.11.017. www.elsevier.com/locate/injury

[2] LeE, J. F. A New Thermal Model for Bone drilling with Applications to Orthopaedic Surgery. Medical Engineering and Physics, 33 (2011), 1234-1244, doi: 10.1016/j.medengphy.2011.05.014.

[3] Hillery, M. T., I. Shuaib. Temperature Effects in the Drilling of Human and Bovine Bone. Journal of Materials Processing Technology, 92-93 (1999), 302-308.

[4] Augustin, G. et Al. Cortical Bone Drilling and Thermal Osteonecrosis. Clin. Biomech., (2011), doi:10.1016/j.clinbiomech.2011.10.010.

[5] Sugano, N. Computer-assisted Orthopedic Surgery. Journal of Orthopaedic Science, 8 (2003), No. 24, 442-448.

[6] Gomes, P. Surgical Robotics: Reviewing the Past, Analysing the Present, Imagining the Future. Robotics and Computer-Integrating Manufacturing, 27 (2011), 261-266.

[7] Beasley, R. A. Medical Robots: Current Systems and Research Directions. Journal of Robotics, Hindawi Publishing Corporation, 2012 (2012), Article ID 40161, doi: $10.1155 / 2012 / 401613$.

[8] Rishi, A. M., R. P. NAYAK. Detection of Breakthrough during Bone drilling in Orthopaedic Surgery. International Journal for Technological Research in Engineering, 1 (2014), No. 9, ISSN (Online): 2347-4718, 794-798. 
[9] Boiadjiev, G. BMM, Orthopaedic Device Useful Model Certificate (Bulgarian Patent), No. 1053, Registry number: 1173, Priority: 2006, Year of publication 2008, 5 (in Bulgarian).

[10] Boindjiev, G. BMM, Orthopedic Device Inventor's Certificate (Bulgarian Patent), No. 66136, Registry number: 109703, Priority: 2008, Year of publication 2011, 7 (in Bulgarian).

[11] Boiadjiev, G., T. Boiadjiev, V. Vitkov, K. Delchev, R. Kastelov, K. ZagursKI. Robotized System for Automation of the Drilling in the Orthopedic Surgery, Control Algorithms and Experimental Results, In Proceedings of the 9th IFAC Symp. on Robot Control SYROCO'09, Japan, Gifu, 2009, 633-638.

[12] Boiadjiev, T., K. Zagurski, G. Boiadjiev, K. Delchev, V. Vitkov, I. Veneva, R. Kastelov. Identification of the Bone Structure during the Automatic Drilling in the Orthopedic Surgery. Journal LMBD Mechanics Based Design of Structures and Machines, 39 (2011), No. 2, 285-302, DOI: 10.1080/15397734.2011.550863.

[13] Boiadjiev, G., R. Kastelov, T. Boiadjiev, V. Kotev, K. Delchev, K. Zagurski, V. Vitkov. Design and Performance Study of an Orthopaedic Surgery Robotized Module for Automatic Bone Drilling. Journal of Medical Robots and Computer Assisted Surgery, 9 (2013), No. 4, 455-463, ISSN 1478596X, Wileyonlinelibrary.com, DOI:10.1002/rcs.1479.

[14] Boiadjiev, T., K. Zagurski, G. Boiadjiev, K. Delchev, R. Kastelov. Automatic Bone Drilling in Orthopedic Surgery. Parameter Tuning of an Active Force Control. Int. J. Applied Mechanics and Materials, 532 (2014), 208-211, Switzerland, Trans. Tech. Publications, ISSN 1660-9336, doi:10.4028 /www.scientific.net/ AMM.532.208

[15] Boiadjiev, G., K. Delchev, T. Boiadjiev, K. Zagurski, R. Kastelov, V. VitKov. Controlled Trust Force Influnce on Automatic Bone Drilling Parameters in the Orthopedic Surgery. Int. J. of Pure and Applied Math., 88 (2013), No. 4, $577-592$

[16] Boiadjiev, T., G. Boiadjiev, K. Delchev, R. Kastelov, K. Zagurski. Eliminating of Far Pedicle Cortex Perforation by Automatic Spine Drilling. Int. Journal of Applied Mechanics and Materials, 799-800 (2015), 505-508, Switzerland, Tech. Publications, doi:10.4028w.scientific.net/ AMM. 799-800.505. ISSN:1660-9336.

[17] Boiadjiev, T., G. Boiadjiev, K. Delchev, R. Kastelov, K. Zagurski. Automatic Bone Drilling in Orthopedic Surgery. Overcoming of the Drill Bit Bending at the Second Cortex. Int. Journal Applied Mechanics and Materials, 664 (2014), 419-422, Switzerland, Trans. Tech. Publications, ISSN 16609336, doi:10.4028 /www.scientific.net/ AMM.644.419 , http://www.ttp.net/ 978-3-03835-292-1.html 